\title{
Higher blood pressure increases arterial stiffness modified by blood glucose levels in a Chinese community-based study
}

This article was published in the following Dove Press journal:

Diabetes, Metabolic Syndrome and Obesity: Targets and Therapy

\author{
Xiaotong Dai' \\ Ying Yang ${ }^{2}$ \\ Guan-Liang Cheng ${ }^{2}$ \\ Jia Jia ${ }^{2}$ \\ Fang-Fang Fan ${ }^{2}$ \\ Jian-Ping $\mathrm{Li}^{2}$ \\ Yong Huo ${ }^{2}$ \\ Zhike Liu' \\ Dafang Chen' \\ Yan Zhang ${ }^{2}$ \\ 'Department of Epidemiology and \\ Biostatistics, School of Public Health, \\ Peking University, Beijing, People's \\ Republic of China; ${ }^{2}$ Department of \\ Cardiology, Peking University First \\ Hospital, Beijing, People's Republic of \\ China
}

Correspondence: Dafang Chen Department of Epidemiology and Biostatistics, School of Public Health, Peking University, No.38, Xueyuan Road, Haidian District, Beijing I0019I, People's

Republic of China

Tel +86 I08 2802644

Fax +86 1082802644

Email dafangchen@bjmu.edu.cn

Yan Zhang

Department of Cardiology, Peking

University First Hospital, No.8 Xishiku St,

Xicheng District, Beijing, People's

Republic of China

Tel +86 1083575728

Email drzhyl108@I63.com
Background: Increased arterial stiffness measured by brachial-ankle pulse wave velocity is associated with cardiovascular disease. However, the rates at which brachial-ankle pulse wave velocity and blood glucose accelerate within individuals who differ in blood pressure levels are largely unknown.

Methods: This study was based on the baseline data of a Chinese community-based atherosclerosis cohort which included 7402 individuals. Using generalized linear regression models, the relationship between blood glucose levels and brachial-ankle pulse wave velocity, and the relationship between blood pressure levels and brachial-ankle pulse wave velocity were examined.

Results: A marked interaction between hypertensive state and diabetic state was seen for brachial-ankle pulse wave velocity ( $P$ for interaction $<0.001$ ). The adjusted coefficient for subjects stratified by hypertensive groups and diabetic states showed that the highest brachial-ankle pulse wave velocity risk subjects were those who had both diabetes and hypertension ( $\mathrm{B}=403.24$; 95\% CI: 372.43-434.05; $P<0.001$ ).

Conclusions: The participants with increased arterial stiffness demonstrate a high prevalence of higher blood pressure. When high blood glucose levels co-occur with high blood pressure levels, there is a remarkable increase in arterial stiffness.

Keywords: communities, diabetes, hypertension

\section{Introduction}

Cardiovascular disease remains a major cause of health loss for all regions of the world. ${ }^{1}$ And epidemiological studies have demonstrated that increased arterial stiffness $^{2,3}$ is an independent risk factor for cardiovascular events. Degenerative stiffness of the arterial beds is referred as arteriosclerosis, ${ }^{4}$ which results in cardiovascular events. An independent predictive value of arterial stiffness for cardiovascular events has been demonstrated in general as well as in selected populations. Increased arterial stiffness is also an independent risk factor for multiple organ damage ${ }^{5}$ and dementia. ${ }^{6,7}$ Arterial stiffness is a shared consequence of diabetes mellitus type 2 (T2DM) and is causally involved in the progression of diabetes complication. ${ }^{8,9}$ On the other hand, cross-sectional studies show that blood pressure (BP) is strongly associated with brachial-ankle pulse wave velocity (baPWV). BP is transmitted into the arterial wall, where its increase progressively stimulates the less distensible collagen fibers, thus resulting in a progressively stiffer artery. ${ }^{10}$ This process may also lead to aortic root dilation. ${ }^{11}$ Therefore, it is important to study arterial stiffness, which can be measured 
by a simple, invasive, and accurate index of brachial-ankle PWV, and it has been clinically validated. ${ }^{12}$

However, the rates at which baPWV and blood glucose accelerate within individuals who differ in blood pressure levels are largely unknown, but their definition is required to unravel the conundrum of interactions of blood glucose levels and blood pressure levels as baPWV increases and is also required to correct power analyses. Therefore, understanding conundrum between blood glucose levels, blood pressure levels, and arterial stiffness is a major public health priority.

To address present gaps in understanding modification of blood glucose levels when the blood pressure level affects arterial stiffness, the relation of a comprehensive panel of blood pressure levels, blood glucose levels was assessed based on the baseline data of a Chinese community-based atherosclerosis cohort.

\section{Materials and methods Study subjects}

The study has been conducted from December 2011 to April 2012 at the baseline of a Chinese community-based atherosclerosis cohort. At first, 9540 participants were recruited in Gucheng and Pingguoyuan communities in Shijingshan district of Beijing, China. The participants were recruited either by responding to recruitment posters that detailed the study or by invited phone calls if they had health medical records in community health centers. The study design and selection criteria have been introduced before. ${ }^{13}$ To avert the bias from the atherosclerosis or related diseases, the patients with a history of coronary heart disease, stroke/ transient ischemic attack, peripheral arterial disease (anklebrachial index $(\mathrm{ABI})<0.9)$, anemia, chronic kidney disease, and renal artery stenosis were excluded $(n=1643)$. Then, missing data were deleted, which included pulse wave velocity (PWV) ( $\mathrm{n}=331$ ), systolic blood pressure (SBP) or diastolic blood pressure (DBP) ( $\mathrm{n}=97)$, fasting blood glucose (FBG) $(n=1), 2-h r$ post-load glucose (2-h PG) $(n=62)$, and ABI $(n=4)$. Finally, 7402 eligible participants were involved in our analyses. All of them offered written informed consent. The study protocol was approved by the ethics committee of Peking University First Hospital. The research was conducted according to the World Medical Association Declaration of Helsinki.

\section{Data collection}

The data were collected by trained research staff according to a standard operation procedure. Subjects were interviewed using a standardized questionnaire, including demographic characteristics, health behavior, histories of drugs and diseases. Current drinking habit was defined as drinking once per week lasting at least half a year. Current smoking habit was defined as current active smokers with one cigarette per day lasting at least half a year. Body mass index (BMI) was calculated as weight $(\mathrm{kg}) /[\text { height }(\mathrm{m})]^{2}$.

\section{Blood pressure tests}

Seated blood pressure was obtained from the right arm with calibrated sphygmomanometer HEM 7117 device (Omron Healthcare Co Ltd) after the subjects had rested in the seated position for 5 mins in a quiet room. The average was calculated using three consecutive measurements separated by $\geq 1$ min interval. In light of 2013 ESCESH guidelines, participants were classified into three groups: optimal $\mathrm{BP}, \quad \mathrm{SBP}<120 \mathrm{mmHg}$, and $\mathrm{DBP}<80$ $\mathrm{mmHg}$ without antihypertensive treatment; prehypertension, SBP 120-139 mmHg, or DBP 80-89 mmHg without antihypertensive treatment; hypertension, $\mathrm{SBP} \geq 140$ $\mathrm{mmHg}$ or $\mathrm{DBP} \geq 90 \mathrm{mmHg}$ or antihypertensive drug usage.

\section{Blood glucose tests}

Overnight fasting blood samples at least $12 \mathrm{hrs}$ were drawn from the antecubital vein using $4 \mathrm{~mL}$ coagulation-promoting vacuum tubes in the morning. Subsequently, the subjects underwent standard 75-g oral glucose tolerance test (or, diabetes mellitus (DM) patients consumed equivalent bread) with $2 \mathrm{hr}$ plasma glucose sampled. Biochemistry index in plasma, including fasting blood glucose, 2-hr post-load glucose, total cholesterol (TC), high-density lipoprotein cholesterol (HDLC), and total triglycerides (TG) were examined by the automatic biochemical analyzer (Roche-C8000) according to a standard procedure. In line with the American Diabetes Association diagnostic criteria, participants were classified into three groups: normal glucose, $\mathrm{FBG}<5.6 \mathrm{mmol} / \mathrm{L}$ and 2-h PG $<7.8 \mathrm{mmol} / \mathrm{L}$ without antidiabetics treatment; prediabetes, FBG $5.6-6.9 \mathrm{mmol} / \mathrm{L}$ or 2 -h PG $7.8-11.0 \mathrm{mmol} / \mathrm{L}$ without antidiabetics treatment; diabetes, $\mathrm{FBG} \geq 7.0 \mathrm{mmol} / \mathrm{L}$ or $2-\mathrm{h} P G \geq 11.1 \mathrm{mmol} / \mathrm{L}$ or any antidiabetics treatment.

\section{Brachial-ankle pulse wave velocity}

After 5 mins seated rest in a quiet room, the brachial-ankle pulse wave velocity (baPWV) measured from the participants in the supine position was obtained by a noninvasive vascular screening device (BP-203RPE II device, Omron Healthcare, Kyoto, Japan) by trained technician according to standard procedures in the morning. Blood pressure and pulse waves velocity in bilateral brachial tibial arteries 
were recorded simultaneously, placing four pneumatic pressure cuff in arms and ankles. The baPWV was the average of left and right baPWV due to the strong correlation between them $(\mathrm{r}=0.968, P<0.001)$.

\section{Statistical analyses}

All data were represented as mean \pm SD for continuous variables and proportion for categorical variables. ANOVA was used to examine intergroup differences of continuous variables. Chi-square test was performed for the comparison of the difference in categorical variables. Generalized linear regression models were applied to assess the association of different blood glucose level and blood pressure level with baPWV. For multiple generalized linear regression models (adjusted models), age, sex, BMI, waist-to-hip ratio (WHR), physical exercise, current drinking, current smoking, TG, TC, high-density lipoprotein cholesterol (HDL), diabetic state, hypertensive groups, antihypertensive drugs, antidiabetics drugs, lipidlowering drugs were adjusted in corresponding models. Moreover, the interaction of diabetic state and hypertensive state on baPWV was detected by the generalized linear regression by adding a cross-product term with main effects and other covariates. Then, the joint effects were estimated after adjusting for covariates. The analyses were performed using SAS 9.3 and Empower(R) (www. empowerstats.com, $\mathrm{X} \& \mathrm{Y}$ solutions, Inc., Boston, MA) and R (http://www.R-project.org). A two-sided $P$-value $<0.05$ was considered statistically significant.

\section{Results}

\section{Characteristics by different blood} pressure level

Characteristics of the study subject by different blood pressure level summarized in Table 1 . The mean age of the

Table I Characteristics by different blood pressure level

\begin{tabular}{|c|c|c|c|c|c|}
\hline Variable & $\begin{array}{l}\text { Total } \\
\mathrm{N}=7402\end{array}$ & Optimal BP $N=|43|$ & $\begin{array}{l}\text { Prehypertension } \\
\mathrm{N}=2697\end{array}$ & $\begin{array}{l}\text { Hypertension } \\
\mathrm{N}=3274\end{array}$ & $P$ \\
\hline Age, yr & $56.04 \pm 8.63$ & $51.92 \pm 7.36$ & $54.82 \pm 7.75$ & $58.84 \pm 8.87$ & $<0.001$ \\
\hline Sex, Male & $2659(35.91)$ & $316(22.08)$ & $1000(37.08)$ & $1342(40.99)$ & $<0.001$ \\
\hline BMI, kg/m² & $25.92 \pm 3.37$ & $24.34 \pm 3.09$ & $25.75 \pm 3.22$ & $26.75 \pm 3.34$ & $<0.001$ \\
\hline WHR, \% & $0.90 \pm 0.06$ & $0.87 \pm 0.06$ & $0.90 \pm 0.06$ & $0.91 \pm 0.06$ & $<0.001$ \\
\hline Waist, cm & $82.40 \pm 8.47$ & $77.87 \pm 7.84$ & $81.95 \pm 8.08$ & $84.75 \pm 8.18$ & $<0.001$ \\
\hline Current smoking & 1449(19.58) & $214(14.95)$ & $597(22.14)$ & $638(19.49)$ & $<0.001$ \\
\hline Current drinking & $|77|(23.93)$ & $25 I(17.54)$ & $684(25.36)$ & $836(25.53)$ & $<0.001$ \\
\hline $\begin{array}{l}\text { Physical exercise, } \\
\geq 5 \text { days per week }\end{array}$ & $4995(67.48)$ & $856(59.82)$ & $1785(66.18)$ & $2354(71.90)$ & $<0.001$ \\
\hline Blood pressure levels & & & & & $<0.001$ \\
\hline Normal glucose & $2980(40.26)$ & $835(58.35)$ & $1210(44.86)$ & $935(28.56)$ & \\
\hline Prediabetes & $2769(37.4 I)$ & $443(30.96)$ & $1007(37.34)$ & $1319(40.29)$ & \\
\hline Diabetes & $1653(22.33)$ & $153(10.69)$ & $480(17.80)$ & $1020(31.15)$ & \\
\hline $\mathrm{TG}, \mathrm{mmol} / \mathrm{L}$ & $1.59 \pm 1.29$ & $1.34 \pm 1.39$ & $1.59 \pm 1.29$ & $1.7 I \pm 1.22$ & $<0.001$ \\
\hline $\mathrm{TC}, \mathrm{mmol} / \mathrm{L}$ & $5.35 \pm 0.99$ & $5.27 \pm 0.96$ & $5.37 \pm 0.99$ & $5.37 \pm 1.01$ & 0.002 \\
\hline $\mathrm{HDL}, \mathrm{mmol} / \mathrm{L}$ & $1.44 \pm 0.38$ & $1.55 \pm 0.40$ & $1.44 \pm 0.38$ & $1.40 \pm 0.36$ & $<0.001$ \\
\hline Antihypertensive drugs & $1972(26.64)$ & 0 & 0 & $1972(60.23)$ & - \\
\hline Lipid-lowering drugs & $495(6.69)$ & $56(3.91)$ & 106(3.93) & $333(10.17)$ & $<0.001$ \\
\hline Antidiabetes drugs & $598(8.08)$ & $65(4.54)$ & $157(5.82)$ & $376(I I .48)$ & - \\
\hline
\end{tabular}

Abbreviations: BMI, body mass index; BP, blood pressure; WHR,waist-to-hip ratio; TG, triglycerides; TC, total cholesterol; HDL, high-density lipoprotein cholesterol. 
population was $56.04 \pm 8.63$ years and $64.09 \%$ of them were women. There was 1431 (19.33\%) normal BP, 2697 (36.44\%) prehypertension, and 3274 (44.23\%) hypertension. Age, sex, BMI, WHR, current smoking, current drinking, physical exercise, hypertensive groups, TG, TC, HDL, antihypertensive drugs, and lipid-lowering drugs were statistically different among the three groups $(P<0.05)$.

\section{The effect of blood pressure levels and blood glucose levels on increased baPWV risk}

The effect of different blood pressure levels and blood glucose levels on increased baPWV risk from generalized linear regression was presented in Figures 1 and 2. In unadjusted model, both prediabetes and diabetes were significantly associated with an increased risk of baPWV compared with normal glucose $(P<0.001)$. The associations were attenuated after adjustment $(\mathrm{B}=43.62$, $P<0.001 ; \mathrm{B}=95.58, P<0.001$, respectively). Similar to the association with diabetic state and baPWV risk, prehypertension and hypertension were significantly associated with an increased risk of baPWV compared with optimal BP $(P<0.001)$ in unadjusted model, and the aforementioned associations were attenuated after adjustment ( $\mathrm{B}=120.34, P<0.001 ; \mathrm{B}=320.35, P<0.001$, respectively). (Table S1.)

\section{The effect of blood glucose levels on the risk of baPWV by different blood pressure level}

As shown in the upper half of Figure 3, there was significantly higher baPWV risk in prediabetes and diabetes compared with normal glucose in all blood pressure levels. Prediabetes did not show a significantly higher baPWV risk compared with normal glucose in optimal BP group $(P=0.459)$ after correcting for confounding variables. (Table S2.)

\section{Interaction of blood glucose levels and blood pressure level on baPWV}

For each blood pressure level, there was a high increase in baPWV with prediabetes and diabetes compared with normal glucose (Figures 4 and 5). When the participants were stratified by blood pressure levels (Table S3.), prediabetes did not have significantly higher baPWV risk for optimal BP group $(P=0.705)$. However, a marked interaction between blood pressure levels and blood glucose levels was seen for baPWV (adjusted $\mathrm{B}=19.71 ; P$ for interaction $<0.001$ ). The adjusted coefficient for subjects stratified by categories of blood pressure levels and blood glucose levels (Table S3.) showed that the highest baPWV risk subjects were those who had both diabetes and hypertension (adjusted $\mathrm{B}=403.24$; $P<0.001$ compared with optimal $\mathrm{BP} /$ normal glucose).

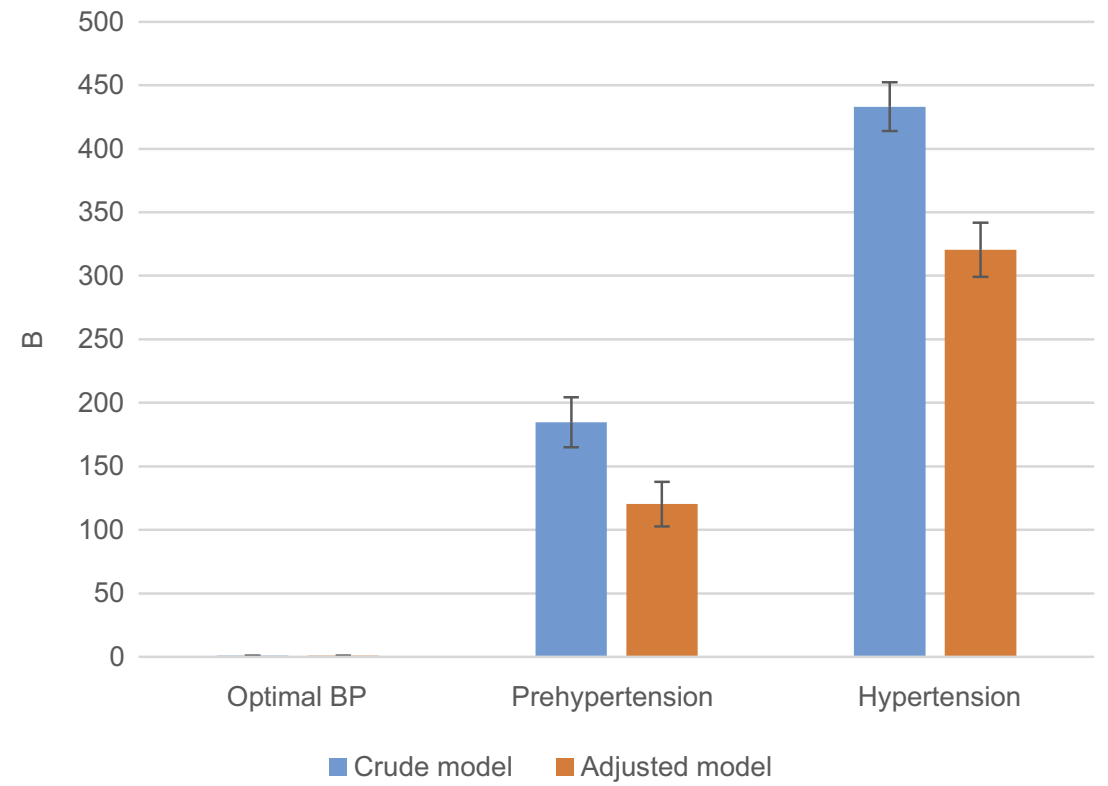

Figure I The effect of blood pressure levels on the risk of baPWV. Adjusted for sex, BMI, WHR, physical exercise, current drinking, current smoking, TG, TC, HDL, antihypertensive drugs, antidiabetes drugs, and lipid-lowering drugs.

Abbreviations: baPWV, brachial-ankle pulse wave velocity; BMI, body mass index; BP, blood pressure; WHR,waist-to-hip ratio; TG, triglycerides; TC, total cholesterol; $\mathrm{HDL}$, high-density lipoprotein cholesterol. 


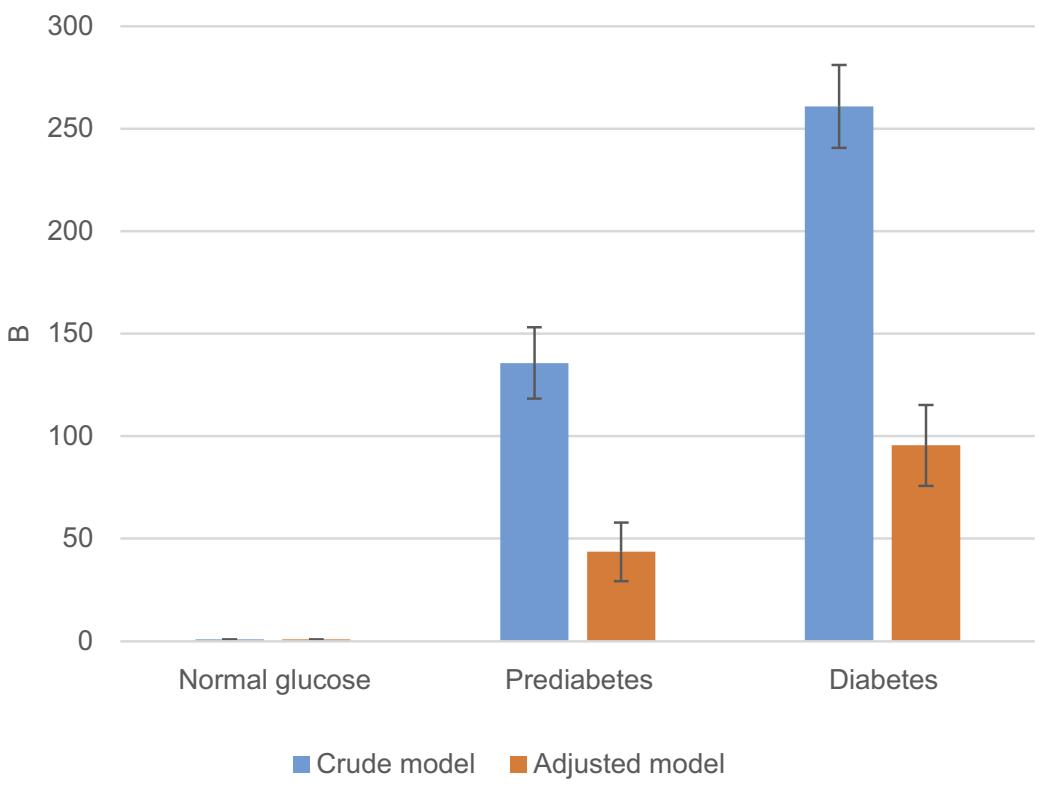

Figure 2 The effect of blood glucose levels on the risk of baPWV. Adjusted for sex, BMI, WHR, physical exercise, current drinking, current smoking, TG, TC, HDL, antihypertensive drugs, antidiabetes drugs, and lipid-lowering drugs.

Abbreviations: baPWV, brachial-ankle pulse wave velocity; BMl, body mass index; WHR,waist-to-hip ratio; TG, triglycerides; TC, total cholesterol; HDL, high-density lipoprotein cholesterol.

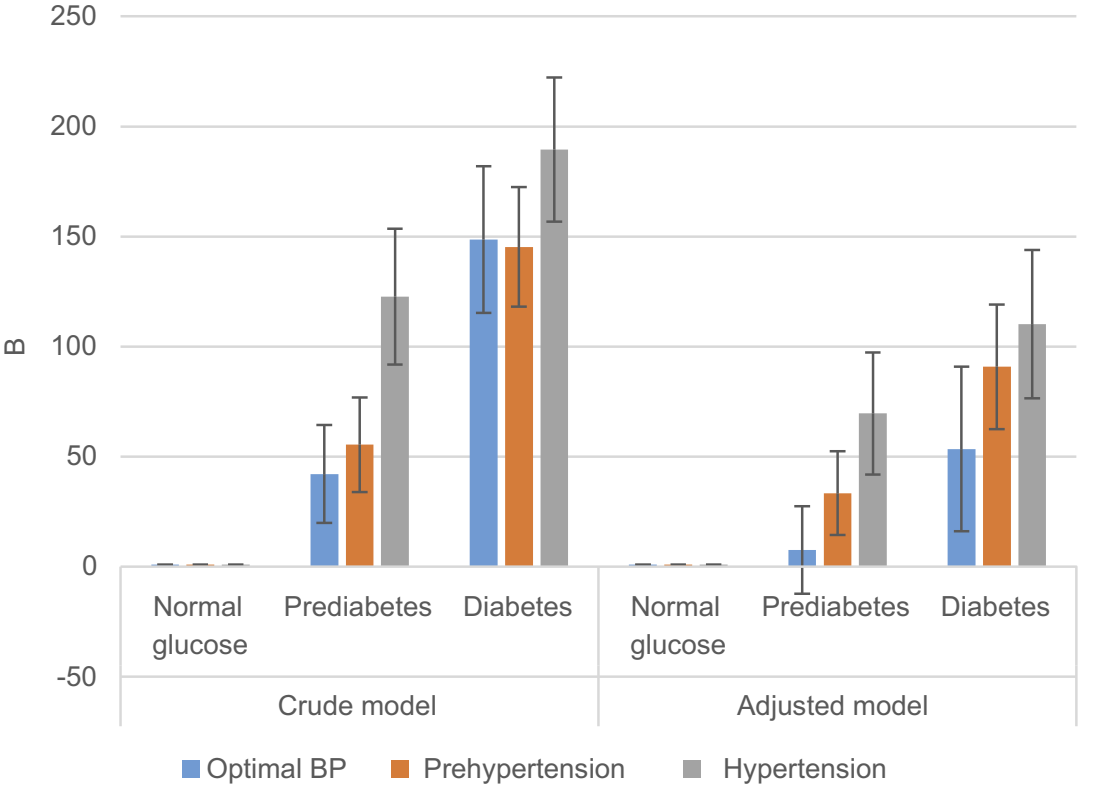

Figure 3 The effect of blood glucose levels on the risk of baPWV by different blood pressure level. Adjusted for sex, BMI, WHR, physical exercise, current drinking, current smoking, TG, TC, HDL, antihypertensive drugs, antidiabetes drugs, and lipid-lowering drugs.

Abbreviations: baPWV, brachial-ankle pulse wave velocity; BMI, body mass index; BP, blood pressure; WHR,waist-to-hip ratio; TG, triglycerides; TC, total cholesterol; HDL, high-density lipoprotein cholesterol.

\section{Discussion}

In this study, we not only observe the association between different blood pressure levels and baPWV, different blood glucose levels and baPWV but also find out the modification of blood glucose levels when higher blood pressure level increases baPWV. To our knowledge, this study is first to show the adverse and independent impact of high blood glucose on arterial stiffness, particularly in those with the higher blood pressure in Chinese community-based population.

Our study shows both prediabetes and diabetes are significantly associated with an increased risk of baPWV compared with normal glucose $(P<0.001)$. And several previous studies 


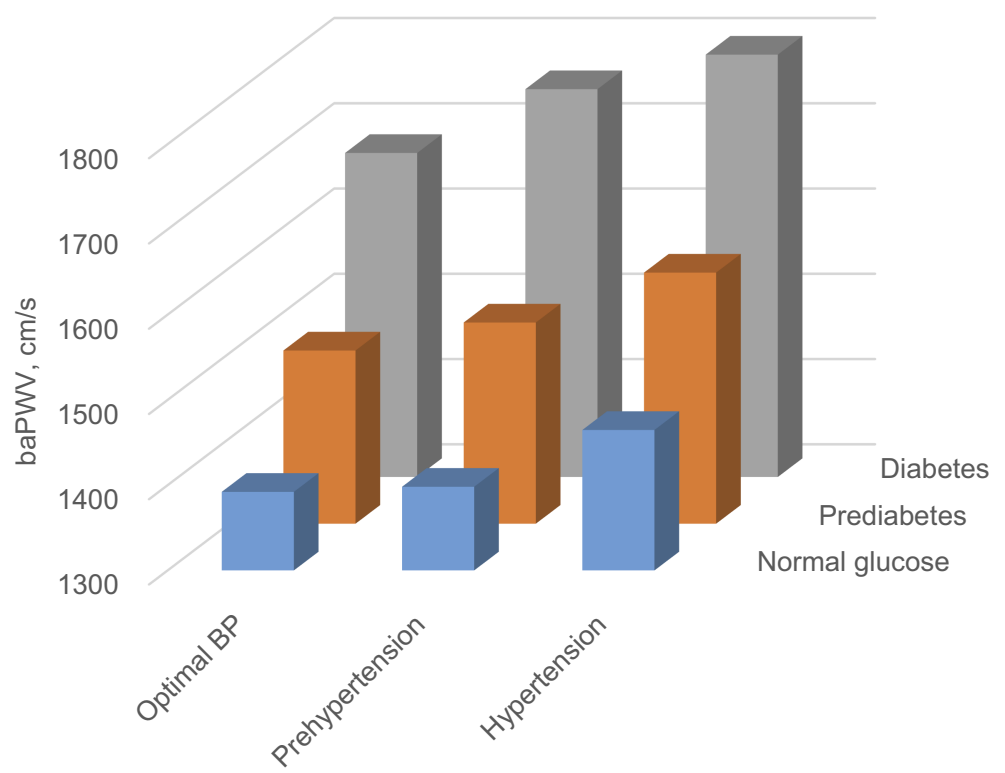

Figure 4 Shown are adjusted least squares mean (standard error) for baPWV by diabetic state and hypertensive groups. Adjusted for sex, BMI, WHR, physical exercise, current drinking, current smoking, TG, TC, HDL, antihypertensive drugs, antidiabetes drugs, and lipid-lowering drugs.

Abbreviations: baPWV, brachial-ankle pulse wave velocity; BMI, body mass index; BP, blood pressure; WHR, waist-to-hip ratio; TG, triglycerides; TC, total cholesterol; HDL, high-density lipoprotein cholesterol.

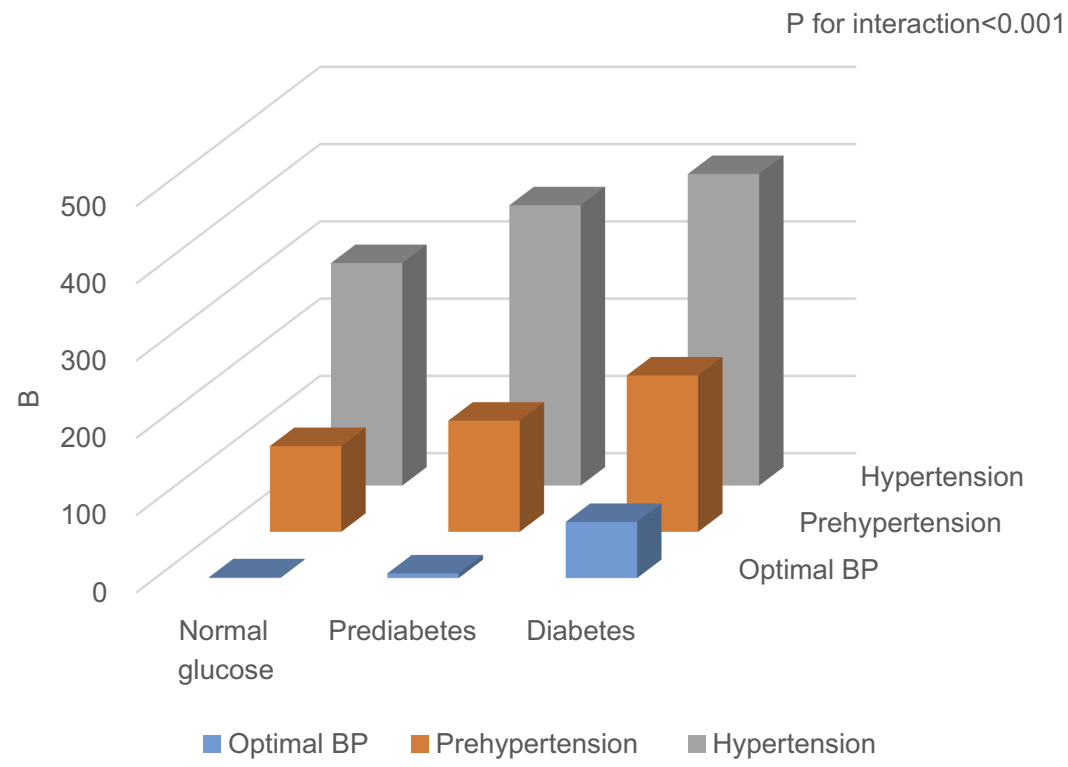

Figure 5 The joint effect and interaction of blood pressure levels, blood glucose levels on baPWV in adjusted models. Adjusted for sex, BMI, WHR, physical exercise, current drinking, current smoking, TG, TC, HDL, antihypertensive drugs, antidiabetes drugs, and lipid-lowering drugs.

Abbreviations: baPWV, brachial-ankle pulse wave velocity; BMI, body mass index; BP, blood pressure; WHR,waist-to-hip ratio; TG, triglycerides; TC, total cholesterol; HDL, high-density lipoprotein cholesterol.

have also shown an increase in fasting plasma glucose (FPG), even within the normal range, associated with aggravated arterial stiffness than the normoglycemia. ${ }^{14-16}$ Prenner et al indicate that one of the most important components in the onset of diabetes is arterial stiffness, and before the development of overt diabetes, the endothelial dysfunction may even be accompanied by the early insulin resistance and impaired fasting glucose. ${ }^{8}$ Another study also finds blunted endothelial function can lead to increased stiffness of the large arteries. ${ }^{17}$ Both endothelial NO dysregulation and advanced glycation end products (AGEs) play huge roles in development of arterial stiffness. ${ }^{8}$ Inhibition of NO bioavailability contributes to the increase in BP. ${ }^{18}$ AGEs is the production of proteins and lipids on exposure to the setting of hyperglycemia. And it can accelerate the age-related vascular changes and the onset of cardiovascular events in both non-diabetic and diabetic 
populations. ${ }^{19-21}$ Furthermore, superoxide production increased and NO synthase activation impaired in the insulinresistant state, leading to a reduction in NO bioavailability. Ultimately it will give rise to arterial stiffness and herald the development of microvascular changes. ${ }^{22-24}$

Another studies release that increased SBP, even in the prehypertension range, relates to the elevated PWV which persisted after adjusting for traditional cardiovascular risk factors. ${ }^{25,26}$ Similar to the result of our research: prehypertension and hypertension were significantly associated with an increased risk of baPWV compared with optimal BP $(P<0.001)$. There is also a complex relationship between arterial stiffness and blood pressure. Some longitudinal studies indicate that higher SBP is associated with faster increase of $\mathrm{PWV},{ }^{25}$ and the results of other reports reveal that higher PWV may herald the longitudinal increase in SBP. $^{27,28}$ The association between arterial stiffness and BP could be described as a vicious cycle. Nigam et al suggest the arterial wall structural alterations and mechanical stretch changes maybe the mechanisms of hypertension on aortic stiffening, such as increased collagen content and decreased elastin content. ${ }^{29}$ Because of the decrease in arterial compliance, the SBP is increased and the DBP is decreased, which contributes to the increase of the pulse pressure (PP). On the one hand, the vascular remodeling impacts $\mathrm{BP}^{30}$ A stiffer wall gives rise to the higher PWV, and then causes the early arrival of reflection pressure waves. The above series of processes lead to the increase in SBP and $\mathrm{PP}^{31}$ Circadian BP variability ${ }^{32}$ and chronic kidney disease also impact on BP variability. ${ }^{33}$ As noted in the Hypertension Branch and Vascular Biology review, the concordance of results in animal and clinical studies strengthens the support for the hypothesis that arterial stiffness plays a potential causal role in the development of hypertension and stresses the need to find molecular pathways that contribute to arterial stiffness and subsequent hypertension. ${ }^{34}$

Epidemiological studies have established that hypertension and DM are independent risk factors for atherosclerosis. However, there are few reports about mechanism of the interaction of blood pressure levels and blood glucose levels on arterial stiffness. Our study releases a marked modification of blood glucose levels when higher blood pressure level increases arterial stiffness ( $P$ for interaction $<0.001$ ). Ma et al indicate that the interaction of hypertension and T2DM aggravates endothelial dysfunction and impairs the smooth muscle function. ${ }^{35}$ Tsao et al demonstrate that both hypertension and DM give rise to increased monocyte adherence to the endothelium. Monocyte chemotactic protein-1 expression and increased vascular superoxide production are associated with the above abnormality. In addition, there may be an additive interaction between hypertension and hyperglycemia in their effects on endothelial adhesiveness. ${ }^{36}$ The inflammation increases in arterial stiffness and thickness. ${ }^{37}$ Smooth muscle cell proliferation, and deterioration of elastin may also result in arterial wall remodeling. ${ }^{38}$

Relatively large sample size, community-based population, and the first attempt to show the modification of blood glucose levels when higher blood pressure level increases arterial stiffness are strengths of this study. However, several limitations should be noted. The limitation of our analysis is its cross-sectional design. We can only find the correlations between variables by regression analysis. More research is needed to reveal the cause-effect relationship. In our work, to avoid excessive collinearity that may make the models robust, we adjusted for some important confounding factors which could increase the arterial stiffness. But we did not control for the phase of the menstrual cycle in premenopausal women, this causes variations in blood pressure and vascular function. And we did not control for caffeine, alcohol consumption or any unaccustomed physical activity $24-\mathrm{h}$ before each visit. This plays a role in vascular function.

\section{Conclusion}

In conclusion, the participants with increased arterial stiffness demonstrated a high prevalence of higher blood glucose, which was independently associated with baPWV. When blood pressure levels co-occurred with blood glucose levels, there was a remarkable increase in baPWV. The findings in our study raised the possibility of the modification of blood glucose levels when higher blood pressure level increases arterial stiffness that, if confirmed in future studies, may have important public health implications for the prevention of arterial stiffness.

\section{Abbreviation list}

baPWV, brachial-ankle pulse wave velocity; T2DM, diabetes mellitus type 2; BP, blood pressure; ABI, anklebrachial index; PWV, pulse wave velocity; SBP, systolic blood pressure; DBP, diastolic blood pressure; FBG, fasting blood glucose; 2-h PG, 2-hr post-load glucose; BMI, Body mass index; DM, diabetes mellitus; TC, total cholesterol; HDL-C/HDL, high-density lipoprotein cholesterol; TG, total triglycerides; WHR, waist-to-hip ratio; AGEs, advanced glycation end products; PP, pulse pressure. 


\section{Ethics approval and consent to participate}

The study protocol was approved by the ethics committee of Peking University First Hospital. The research was conducted according to the World Medical Association Declaration of Helsinki. The adolescents who participated in this study agreed in writing to participate and their legal guardians signed an informed consent form.

\section{Availability of data and materials}

The data that support the findings of this study are available from Peking University First Hospital, but restrictions apply to the availability of these data, which were used under license for the current study, and so are not publicly available. Data are however available from the authors upon reasonable request and with permission of Peking University First Hospital.

\section{Acknowledgments}

We really appreciate the staff of Gucheng and Pingguoyuan Community Health Centers and their research coordinators who participated in this survey and thank them for their assistance in data acquisition.

The study was supported by grants from the National Natural Science Foundation of China (81172768, 81872692), Beijing Municipal Natural Science Foundation (7182085) and the UM-PUHSC Joint Institute for Translational and Clinical Research (BMU20110177).

\section{Author contributions}

$X$ Dai wrote the manuscript. Z Liu performed the statistical analyses. D Chen and Y Zhang revised the paper. All authors contributed towards data analysis, drafting and critically revising the paper, gave final approval of the version to be published, and agreed to be accountable for all aspects of the work.

\section{Disclosure}

The authors declare that they have no competing interests in this work.

\section{References}

1. Roth GA, Johnson C, Abajobir A. Global, regional, and national burden of cardiovascular diseases for 10 causes, 1990 to 2015. $\mathrm{J} \mathrm{Am}$ Coll Cardiol. 2017;70(1):1-25. doi:10.1016/j.jacc.2017.04.052

2. Cui R, Yamagishi K, Muraki I. Investigators. C. Association between markers of arterial stiffness and atrial fibrillation in the circulatory risk in communities study (CIRCS). Atherosclerosis. 2017;263:244-248. doi:10.1016/j.atherosclerosis.2017.06.918
3. Ohyama Y, Ambale-Venkatesh B, Noda C. Aortic arch pulse wave velocity assessed by magnetic resonance imaging as a predictor of incident cardiovascular events: the MESA (Multi-ethnic study of atherosclerosis). Hypertension. 2017;70:524-530. doi:10.1161/ HYPERTENSIONAHA.116.08749

4. Cavalcante JL, Lima JA, Redheuil A, Al-Mallah MH. Aortic stiffness: current understanding and future directions. $\mathrm{J} \mathrm{Am} \mathrm{Coll} \mathrm{Cardiol.}$ 2011;57(14):1511-1522. doi:10.1016/j.jacc.2010.12.017

5. Scuteri A, Rovella V, Alunni FD, Tesauro M, Gabriele M, Di Daniele N. An operational definition of SHATS (Systemic hemodynamic atherosclerotic syndrome): role of arterial stiffness and blood pressure variability in elderly hypertensive subjects. Int $J$ Cardiol. 2018;263:132-137. doi:10.1016/j.ijcard.2018.03.117

6. Scuteri A, Tesauro M, Guglini L, Lauro D, Fini M, Di Daniele N. Aortic stiffness and hypotension episodes are associated with impaired cognitive function in older subjects with subjective complaints of memory loss. Int $J$ Cardiol. 2013;169(5):371-377. doi:10.1016/j.ijcard.2013.09.009

7. Scuteri A, Wang H. Pulse wave velocity as a marker of cognitive impairment in the elderly. J Alzheimers Dis. 2014;42(Suppl 4):S401S410. doi:10.3233/JAD-141416

8. Prenner SB, Chirinos JA. Arterial stiffness in diabetes mellitus. Atherosclerosis. 2015;238(2):370-379. doi:10.1016/j.atheroscleros is. 2014.12 .023

9. Xu M, Huang Y, Xie L, et al. Diabetes and risk of arterial stiffness: a mendelian randomization analysis. Diabetes. 2016;65(6):1731-1740. doi:10.2337/db15-1533

10. Scuteri A, Morrell CH, Orru M, et al. Longitudinal perspective on the conundrum of central arterial stiffness, blood pressure, and aging. Hypertension. 2014;64(6):1219-1227. doi:10.1161/ HYPERTENSIONAHA.114.04127

11. Farasat SM, Morrell CH, Scuteri A, et al. Pulse pressure is inversely related to aortic root diameter implications for the pathogenesis of systolic hypertension. Hypertension. 2008;51(2):196-202. doi:10.1161/HYPERTENSIONAHA.107.099515

12. Sugawara J, Tanaka H. Brachial-ankle pulse wave velocity: myths, misconceptions, and realities. Pulse (Basel). 2015;3(2):106-113. doi:10.1159/000430771

13. Fan F, Qi L, Jia J, et al. Noninvasive central systolic blood pressure is more strongly related to kidney function decline than peripheral systolic blood pressure in a Chinese community-based population. Hypertension. 2016;67(6):1166-1172. doi:10.1161/HYPERTEN SIONAHA.115.07019

14. Paik JK, Kim M, Kwak JH, Lee EK, Lee SH, Lee JH. Increased arterial stiffness in subjects with impaired fasting glucose. $J$ Diabetes Complications. 2013;27(3):224-228. doi:10.1016/j. jdiacomp.2012.10.012

15. Shin JY, Lee HR, Lee DC. Increased arterial stiffness in healthy subjects with high-normal glucose levels and in subjects with pre-diabetes. Cardiovasc Diabetol. 2011;10:30. doi:10.1186/1475-2840-10-30

16. Rahman S, Ismail AA, Ismail SB, Naing NN, Rahman AR. Early manifestation of macrovasculopathy in newly diagnosed never treated type II diabetic patients with no traditional CVD risk factors. Diabetes Res Clin Pract. 2008;80(2):253-258. doi:10.1016/j.diabres.2007.12.010

17. Scuteri A, Tesauro M, Rizza S, et al. Endothelial function and arterial stiffness in normotensive normoglycemic first-degree relatives of diabetic patients are independent of the metabolic syndrome. Nutr Metab Cardiovasc Dis. 2008;18(5):349-356. doi:10.1016/j. numecd.2007.03.008

18. Scuteri A, Stuehlinger MC, Cooke JP, et al. Nitric oxide inhibition as a mechanism for blood pressure increase during salt loading in normotensive postmenopausal women. $J$ Hypertens. 2003;21 (7):1339-1346. doi:10.1097/01.hjh.0000059082.43904.02

19. Sell DR, Monnier VM. Molecular basis of arterial stiffening: role of glycation - a mini-review. Gerontology. 2012;58(3):227-237. doi: $10.1159 / 000334668$ 
20. Kilhovd BK, Juutilainen A, Lehto S, et al. Increased serum levels of advanced glycation endproducts predict total, cardiovascular and coronary mortality in women with type 2 diabetes: a population-based 18 year follow-up study. Diabetologia. 2007;50 (7):1409-1417. doi:10.1007/s00125-007-0687-z

21. Nin JW, Jorsal A, Ferreira I, et al. Higher plasma levels of advanced glycation end products are associated with incident cardiovascular disease and all-cause mortality in type 1 diabetes: a 12-year follow-up study. Diabetes Care. 2011;34(2):442-447. doi:10.2337/dc10-1087

22. Du X, Edelstein D, Obici S, Higham N, Zou MH, Brownlee M. Insulin resistance reduces arterial prostacyclin synthase and eNOS activities by increasing endothelial fatty acid oxidation. J Clin Invest. 2006;116(4):1071-1080. doi:10.1172/JCI23354

23. Paneni F, Beckman JA, Creager MA, Cosentino F. Diabetes and vascular disease: pathophysiology, clinical consequences, and medical therapy: part I. Eur Heart J. 2013;34(31):2436-2443. doi:10.1093/eurheartj/eht149

24. Brillante DG, O'Sullivan AJ, Howes LG. Arterial stiffness in insulin resistance: the role of nitric oxide and angiotensin II receptors. Vasc Health Risk Manag. 2009;5(1):73-78.

25. AlGhatrif M, Strait JB, Morrell CH, et al. Longitudinal trajectories of arterial stiffness and the role of blood pressure: the Baltimore longitudinal study of aging. Hypertension. 2013;62(5):934-941. doi:10.1161/HYPERTENSIONAHA.113.01445

26. Lim J, Pearman ME, Park W, Alkatan M, Machin DR, Tanaka H. Impact of blood pressure perturbations on arterial stiffness. Am J Physiol Regul Integr Comp Physiol. 2015;309(12):R1540-R1545. doi:10.1152/ajpregu.00368.2015

27. Najjar SS, Scuteri A, Shetty V, et al. Pulse wave velocity is an independent predictor of the longitudinal increase in systolic blood pressure and of incident hypertension in the Baltimore longitudinal study of aging. J Am Coll Cardiol. 2008;51(14):1377-1383. doi:10.1016/j.jacc.2007.10.065

28. Kaess BM, Rong J, Larson MG, et al. Aortic stiffness, blood pressure progression, and incident hypertension. Jama. 2012;308(9):875-881. doi:10.1001/2012.jama.10503
29. Nigam A, Mitchell GF, Lambert J, Tardif JC. Relation between conduit vessel stiffness (assessed by tonometry) and endothelial function (assessed by flow-mediated dilatation) in patients with and without coronary heart disease. Am J Cardiol. 2003;92(4):395-399.

30. Scuteri A, Chen CH, Yin FC, Chih-Tai T, Spurgeon HA, Lakatta EG Functional correlates of central arterial geometric phenotypes. Hypertension. 2001;38(6):1471-1475.

31. Vulliemoz S, Stergiopulos N, Meuli R. Estimation of local aortic elastic properties with MRI. Magn Reson Med. 2002;47 (4):649-654.

32. Scuteri A, Morrell CH, Orru' M, et al. Gender specific profiles of white coat and masked hypertension impacts on arterial structure and function in the SardiNIA study. Int J Cardiol. 2016;217:92-98. doi:10.1016/j.ijcard.2016.04.172

33. Di Daniele N, Fegatelli DA, Rovella V, Castagnola V, Gabriele M, Scuteri A. Circadian blood pressure patterns and blood pressure control in patients with chronic kidney disease. Atherosclerosis. 2017;267:139-145. doi:10.1016/j.atherosclerosis.2017.10.031

34. Galis ZS, Thrasher T, Reid DM, Stanley DV, Oh YS. Investing in high blood pressure research: a national institutes of health perspective. Hypertension. 2013;61(4):757-761. doi:10.1161/ HYPERTENSIONAHA. 111.00770

35. Ma L, Zhao S, Li J, Zhou Q, Gao M. Interaction of hypertension and diabetes on impairment of endothelial function. Chin Med J (Engl). 2001;114(6):563-567.

36. Tsao PS, Niebauer J, Buitrago R, et al. Interaction of diabetes and hypertension on determinants of endothelial adhesiveness. Arterioscler Thromb Vasc Biol. 1998;18(6):947-953.

37. Scuteri A, Orru M, Morrell C, et al. Independent and additive effects of cytokine patterns and the metabolic syndrome on arterial aging in the SardiNIA study. Atherosclerosis. 2011;215(2):459-464. doi:10.1016/j.atherosclerosis.2010.12.023

38. Ganguly P, Alam SF. Role of homocysteine in the development of cardiovascular disease. Nutr J. 2015;14:6. doi:10.1186/1475-289114-6 


\section{Supplementary materials}

Table SI The effect of blood pressure levels, blood glucose levels on the risk of baPWV

\begin{tabular}{|c|c|c|c|c|}
\hline \multirow[t]{2}{*}{ Variables } & \multicolumn{2}{|l|}{ Crude model } & \multicolumn{2}{|l|}{ Adjusted model } \\
\hline & B(95\%CI) & $P$ & $\mathrm{~B}(95 \% \mathrm{Cl})$ & $P$ \\
\hline $\begin{array}{l}\text { Blood pressure levels } \\
\text { Optimal BP } \\
\text { Prehypertension } \\
\text { Hypertension }\end{array}$ & $\begin{array}{l}\text { Ref } \\
\mid 84.65(|64.88,204.4|) \\
433.0 \mid(4 \mid 3.86,452.16)\end{array}$ & $\begin{array}{l}<0.001 \\
<0.001\end{array}$ & $\begin{array}{l}\text { Ref } \\
\text { I20.34(|02.74,| } 37.93) \\
320.35(299.0|, 34| .69)\end{array}$ & $\begin{array}{l}<0.001 \\
<0.001\end{array}$ \\
\hline $\begin{array}{l}\text { Blood glucose levels } \\
\text { Normal glucose } \\
\text { Prediabetes } \\
\text { Diabetes }\end{array}$ & $\begin{array}{l}\text { Ref } \\
\mid 35.72(|| 8.3|,| 53 . \mid 3) \\
260.78(240.55,28|.0|)\end{array}$ & $\begin{array}{l}<0.001 \\
<0.001\end{array}$ & $\begin{array}{l}\text { Ref } \\
43.62(29.33,57.90) \\
95.58(75.85,115.32)\end{array}$ & $\begin{array}{l}<0.001 \\
<0.001\end{array}$ \\
\hline
\end{tabular}

Abbreviation: baPWV, brachial-ankle pulse wave velocity.

Table S2 The effect of blood glucose levels on the risk of baPWV by different blood pressure level

\begin{tabular}{|c|c|c|c|c|c|c|}
\hline \multirow[t]{2}{*}{ Blood glucose levels } & \multicolumn{2}{|l|}{ Optimal BP } & \multicolumn{2}{|l|}{ Prehypertension } & \multicolumn{2}{|l|}{ Hypertension } \\
\hline & $\mathrm{B}(95 \% \mathrm{Cl})$ & $P$ & $\mathbf{B}(95 \% \mathrm{CI})$ & $P$ & $\mathbf{B}(95 \% \mathrm{Cl})$ & $P$ \\
\hline $\begin{array}{l}\text { Unadjusted model } \\
\text { Normal glucose } \\
\text { Prediabetes } \\
\text { Diabetes }\end{array}$ & $\begin{array}{l}\text { Ref } \\
42.12(\mid 9.83,64.40) \\
|48.6|(|15.27,| 8 \mid .95)\end{array}$ & $\begin{array}{l}0.002 \\
<0.001\end{array}$ & $\begin{array}{l}\text { Ref } \\
55.46(33.96,76.97) \\
\mid 45.28(|||8.08,| 72.48)\end{array}$ & $\begin{array}{l}<0.001 \\
<0.001\end{array}$ & $\begin{array}{l}\text { Ref } \\
\mid 22.63(9|.79,| 53.47) \\
|89.5|(\mid 56.85,222.17)\end{array}$ & $\begin{array}{l}<0.001 \\
<0.001\end{array}$ \\
\hline $\begin{array}{l}\text { Adjusted model } \\
\text { Normal glucose } \\
\text { Prediabetes } \\
\text { Diabetes }\end{array}$ & $\begin{array}{l}\text { Ref } \\
7.55(-12.30,27.4 I) \\
53.48(16.13,90.83)\end{array}$ & $\begin{array}{l}0.459 \\
0.005\end{array}$ & $\begin{array}{l}\text { Ref } \\
33.42(14.38,52.46) \\
90.86(62.57,119.15)\end{array}$ & $\begin{array}{l}<0.001 \\
<0.001\end{array}$ & $\begin{array}{l}\text { Ref } \\
69.6(4 \mid .85,97.35) \\
110.16(76.45, \mid 43.88)\end{array}$ & $\begin{array}{l}<0.001 \\
<0.001\end{array}$ \\
\hline
\end{tabular}

Abbreviations: baPWV, brachial-ankle pulse wave velocity; $\mathrm{BP}$, blood pressure.

Table S3 Interaction of blood pressure levels, blood glucose levels on baPWV

\begin{tabular}{|c|c|c|c|c|c|}
\hline \multirow[t]{2}{*}{ Blood pressure levels } & \multirow[t]{2}{*}{ Blood glucose levels } & \multicolumn{2}{|l|}{ Unadjusted Model } & \multicolumn{2}{|l|}{ Adjusted Model } \\
\hline & & $\mathbf{B}(95 \% \mathrm{Cl})$ & $P$ & $\mathbf{B}(95 \% \mathrm{CI})$ & $\boldsymbol{P}$ \\
\hline Optimal BP & Normal glucose & Ref & & Ref & \\
\hline Optimal BP & Prediabetes & $42.12(7.32,76.92)$ & 0.018 & $5.96(-24.92,36.84)$ & 0.705 \\
\hline Optimal BP & Diabetes & $|48.6|(96.54,200.68)$ & $<0.001$ & $72.58(24.96,120.21)$ & 0.003 \\
\hline Prehypertension & Normal glucose & $167.01(140.37,193.65)$ & $<0.001$ & III.0I(87.|14,134.89) & $<0.001$ \\
\hline Prehypertension & Prediabetes & $222.48(194.76,250.19)$ & $<0.001$ & $143.84(\mid 18.58,169.10)$ & $<0.001$ \\
\hline Prehypertension & Diabetes & $312.29(278.38,346.20)$ & $<0.001$ & $202.36(170.02,234.70)$ & $<0.001$ \\
\hline Hypertension & Normal glucose & $353.49(325.30,381.69)$ & $<0.001$ & $287.95(260.16,315.73)$ & $<0.001$ \\
\hline Hypertension & Prediabetes & $476.12(449.94,502.31)$ & $<0.001$ & $362.76(335.50,390.02)$ & $<0.001$ \\
\hline Hypertension & Diabetes & $543(515.37,570.64)$ & $<0.001$ & $403.24(372.43,434.05)$ & $<0.001$ \\
\hline Interaction & & $21.15(8.79,33.5 \mathrm{I})$ & $<0.001$ & $|9.7|(8.68,30.73)$ & $<0.001$ \\
\hline
\end{tabular}

Abbreviation: baPWV, brachial-ankle pulse wave velocity. 
Table S4 Interaction of physical exercise and the combined variable of blood pressure levels and blood glucose levels on baPWV

\begin{tabular}{|l|l|l|l|}
\hline Blood pressure levels & Blood glucose levels & $\begin{array}{l}\text { Inactive participants } \\
\mathbf{B}(\mathbf{9 5} \% \mathbf{C I})\end{array}$ & $\begin{array}{l}\text { Sedentary participants } \\
\mathbf{B}(\mathbf{9 5} \% \mathbf{C I})\end{array}$ \\
\hline Optimal BP & Normal glucose & Ref & Ref \\
Optimal BP & Prediabetes & $13.69(-30.82,58.19)$ & $0.31(-41.05,41.66)$ \\
Optimal BP & Diabetes & $49.67(-21.73,121.06)$ & $82.35(20.06,144.64)$ \\
Prehypertension & Normal glucose & $113.53(79.10,147.95)$ & $108.90(76.87,140.92)$ \\
Prehypertension & Prediabetes & $139.80(173.56,275.59)$ & $145.58(111.92,179.25)$ \\
Prehypertension & Diabetes & $224.58(219.96,302.99)$ & $193.12(151.61,234.62)$ \\
Hypertension & Normal glucose & $261.47(281.07,365.38)$ & $300.47(263.91,337.03)$ \\
Hypertension & Prediabetes & $323.23(301.72,344.73)$ & $377.40(342.01,412.80)$ \\
Hypertension & Diabetes & $380.57(331.54,429.61)$ & $410.17(370.66,449.69)$ \\
P for Interaction & \multicolumn{2}{|l}{} & \\
\hline
\end{tabular}

Abbreviations: baPWV, brachial-ankle pulse wave velocity; BP, blood pressure.

\section{Publish your work in this journal}

Diabetes, Metabolic Syndrome and Obesity: Targets and Therapy is an international, peer-reviewed open-access journal committed to the rapid publication of the latest laboratory and clinical findings in the fields of diabetes, metabolic syndrome and obesity research. Original research, review, case reports, hypothesis formation, expert opinion and commentaries are all considered for publication. The manuscript management system is completely online and includes a very quick and fair peer-review system, which is all easy to use. Visit $\mathrm{http}: / /$ www.dovepress.com/testimonials.php to read real quotes from published authors. 